\title{
Refractory period of single motor nerve fibres in man
}

\author{
JÖRGEN BORG
}

From the Department of Neurology, Karolinska Hospital, Stockholm, Sweden

SUMMARY Electromyographic single motor unit recordings were used to study the axonal refractory period of 28 accessory nerve innervated motor units in young normal subjects and 10 reinnervated motor units in elderly subjects. The refractory period was measured after a conditioning antidromic propagated nerve impulse and after a conditioning electrical nerve stimulus delivered through the same electrode as the test stimulus. The refractory period after a propagated impulse was $1.83 \pm 0.26 \mathrm{~ms}$ when test stimulus strength was $10 \%$ above the axonal threshold at rest and $0 \cdot 88 \pm 0 \cdot 26 \mathrm{~ms}$ when test stimulus strength was $100 \%$ above threshold. Corresponding data after an electrical stimulus were $2.38 \pm 0.32 \mathrm{~ms}$ and $1.28 \pm 0.22 \mathrm{~ms}$. The difference between the methods was partially due to the effect of stimulus spread when the refractory period was measured after an antidromic propagated nerve impulse. Different properties of the nerve fibre membrane and the surrounding tissues after a propagated nerve impulse and after an electrical stimulus might also contribute. For further studies of motor unit pathology both methods seem equally reliable when proper normal data are available.

In animal experiments the refractory period of a single nerve fibre is measured by delivering a conditioning and a testing electrical stimulus through the same electrode to the nerve fibre and determining the excitability changes after the first, conditioning, stimulus.' In man this technique has been applied to whole nerve trunks containing different fibre types. $^{23}$ To measure the refractory period of motor nerve fibres, electromyographic recordings are required and a blocking impulse is necessary to avoid the overlapping of the recorded, compound muscle action potentials, as described by Kimura. ${ }^{4}$

In a previous study of single alpha motor axons in man the axonal refractory period was determined after an antidromic propagated nerve impulse. ${ }^{5}$ The aim of that study was to determine how to correct for the refractory period when antidromic propagated nerve impulses are used to measure the conduction velocity spectrum of peripheral motor nerves in man as described by Hopf. ${ }^{\circ}$

In the present study the axonal refractory period was determined for single short toe extensor motor units both after a conditioning antidromic propagated impulse and after a conditioning electrical

Address for reprint requests: Dr Jörgen Borg, Department of Neurology, Karolinska Hospital, S-104 01 Stockholm, Sweden.

Received 19 July 1983 and in revised form 17 October 1983. Accepted 5 November 1983 nerve stimulus. This was possible since it was found that after delivering paired electrical stimuli proximal to the peroneal nerve, the slowing of the conduction of the second impulse while propagated during the relative refractory period of the prior impulse prevents blockings of the neuro-muscular transmission even at short stimulus intervals. ${ }^{7}$ Thus, the axonal refractory period can be measured at a proximal test point after a conditioning electrical stimulus without using blocking impulses. The aim of the present study was to compare the results obtained by each method in normal subjects.

\section{Materials and methods}

The study included seven subjects aged 24-36 years, all with an accessory deep peroneal nerve innervating just a few motor units, and three subjects aged 74-78 years. All were healthy and had normal maximum conduction velocities.

Electromyographic recordings were made from the short toe extensor muscle by conventional bipolar needle electrodes (DISA 9013 K0802 Electronic, Skovlunde, Denmark). Selective recordings permitting identification of single motor unit potentials at maximum voluntary contraction and after supramaximum nerve stimulation were achieved (1) from motor units innervated by the accessory peroneal nerve after blocking of the main nerve with local anaesthesia, ${ }^{8}$ (2) from motor units in elderly subjects in whom loss of motor units and increased fibre density of the remaining ones normally occurs. ${ }^{9}$

The motor unit potentials were amplified and displayed 
on a Medelec oscilloscope No 4329 and recorded on Kodak Linagraph direct print paper. The common peroneal nerve was stimulated proximally at the fibular head, and distally at the ankle, posterior or anterior to the lateral malleolus. Stimuli were delivered through surface electrodes $0.6 \mathrm{~cm}$ in diameter. The cathode was placed over the nerve and the anode $2-3 \mathrm{~cm}$ laterally or medially to the cathode. Rectangular pulse waves of $0.2 \mathrm{~ms}$ duration were used. Stimulus strength could be gradually changed from 0 to $100 \mathrm{~mA}$. After adjusting the electrode so that the test motor unit potential could be safely identified in sustained maximum voluntary contraction, the effort was reduced until the motor unit no longer discharged and electrical stimuli were delivered in each of the following ways: (1) A single nerve stimulus was delivered proximally and then distally, the latency difference was calculated and the axonal conduction velocity determined.

(2) Paired stimuli were delivered through the same electrode to the nerve at the proximal stimulus point. The stimulus strength was initially $10 \%$ above the axonal threshold at rest for both the first, conditioning, and the second, testing, stimulus. The stimulus interval was initially $5 \mathrm{~ms}$ and then reduced by $0.2 \mathrm{~ms}$ intervals until the second evoked motor unit potential disappeared. This was due to blocking at the stimulus point during the refractory period after the conditioning stimulus, because when test stimulus strength increased the second evoked potential reappeared. By determining the shortest stimulus interval without blocking, the axonal refractory period following a conditioning electrical nerve stimulus could be determined.'

(3) One single proximal and one single distal stimulus were then delivered coupled to each other. The strength of both stimuli was initially $10 \%$ above the axonal threshold at rest. The proximal stimulus was initially delayed 10-15 $\mathrm{ms}$ to permit the distally evoked, antidromic propagated nerve impulse to pass the proximal test point before stimulation. The stimulus interval was then reduced by $0.2 \mathrm{~ms}$ intervals until the second evoked motor unit potential disappeared. This was due to blocking at the proximal, testing, point during the refractory period after the antidromic impulse, because when test stimulus strength increased the second motor unit potential reappeared. By comparing the shortest stimulus interval without blocking with the previously determined latency difference (point 1 above) the axonal refractory period following an antidromic propagated nerve impulse was calculated. ${ }^{5}$ The strength of the proximal stimulus was the same when the latency difference and the shortest stimulus interval without blocking were determined to avoid errors caused by stimulus spread.

Room temperature was $23-25^{\circ} \mathrm{C}$. Skin temperature was held at $32^{\circ}$ by a DISAheater (DISA Electronic, Skovlunde, Denmark).

\section{Results}

For 38 motor units the axonal refractory period was determined after a conditioning electrical nerve stimulus and after a conditioning antidromic propagated nerve impulse using low test stimulus strength, that is, $10 \%$ above the axonal threshold at rest. Figure 1 shows the recordings from such an experiment. Figure $1 \mathrm{~A}$ shows the recordings after a single distal nerve stimulus and fig $1 \mathrm{~B}$ after a single proximal nerve stimulus. The latency difference was $10 \cdot 0 \mathrm{~ms}$. The conduction distance was $40 \mathrm{~cm}$ and thus the axonal conduction velocity for this motor unit was $40 \mathrm{~m} / \mathrm{s}$. Figure $1 \mathrm{C}$ shows the recordings after paired electrical stimuli were delivered at the proximal stimulus point with a stimulus interval of $2.2 \mathrm{~ms}$. The second evoked motor unit response was delayed in relation to the second stimulus due to slowing of the conduction of the second impulse propagated during the relative refractory period of the prior impulse. ${ }^{7}$ Figure 1D shows that when the stimulus interval was reduced to $2.0 \mathrm{~ms}$, the second motor unit response disappeared. This was due to refractoriness after the first, conditioning stimulus at the test point because when test stimulus strength increased the second motor unit response reoccurred. Figure $1 \mathrm{E}$ shows the recordings when one single distal and one single proximal stimulus were delivered coupled to each other with the distal stimulus preceding the proximal by $11.6 \mathrm{~ms}$. Figure $1 \mathrm{~F}$ shows that when the stimulus interval was reduced to 11.4 $\mathrm{ms}$ the proximally evoked motor unit response disappeared. This was due to refractoriness after the antidromic propagated nerve impulse at the test point because when the proximal, testing stimulus strength increased the proximally evoked motor unit response reoccurred. Thus, the calculated refractory period after an antidromic impulse was $1.6 \mathrm{~ms}$ (11.6-10.0).

For 28 motor units in seven young subjects and 10 motor units in three elderly subjects with axonal conduction velocities $32-46 \mathrm{~m} / \mathrm{s}$, the axonal refractory period was determined as described in fig 1 . One to seven motor units were studied in each subject. For ten accessory motor nerve motor units with axonal conduction velocities $34-43 \mathrm{~m} / \mathrm{s}$ the refractory period was also determined using high test stimulus strength, that is $100 \%$ above the axonal threshold. One to three motor units were studied in each subject.

\section{REFRACTORY PERIOD AFTER A CONDITIONING}

\section{ELECTRICAL NERVE STIMULUS}

When test stimulus strength was low the refractory period ranged from 1.8 to $3.3 \mathrm{~ms}$. In only one motor unit it was longer than $3 \mathrm{~ms}$ and in only two motor units it was shorter than $2 \mathrm{~ms}$. The mean value for all motor units was $2 \cdot 34 \pm 0.32 \mathrm{~ms}(\mathrm{M} \pm \mathrm{SD})$ and for the 10 individual mean values $2.38 \pm 0.24 \mathrm{~ms}$. There was no difference between the age groups.

When test stimulus strength was high the refractory period ranged from 1.0 to $1.6 \mathrm{~ms}$. The mean 

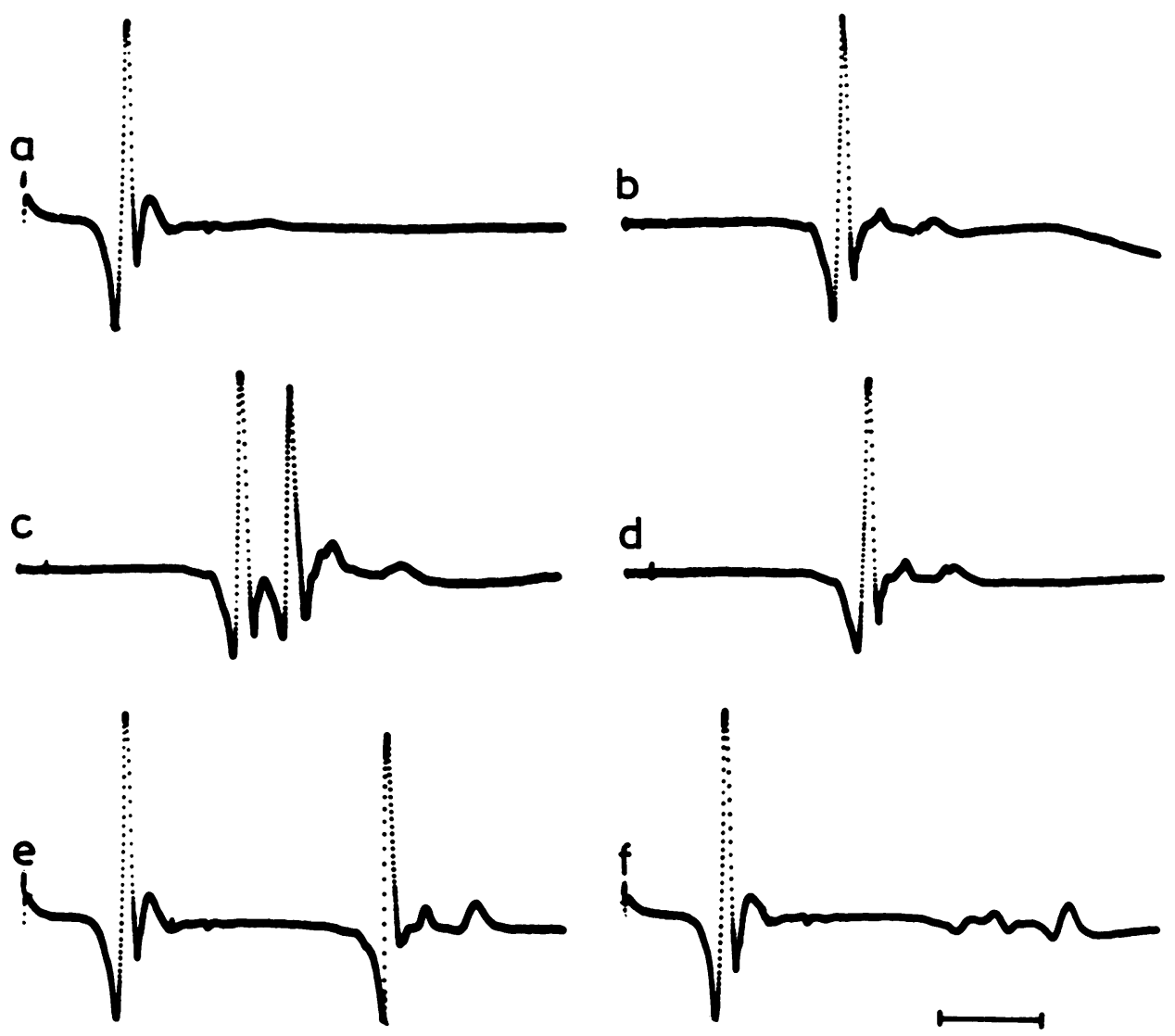

Fig 1 Electromyographic single motor unit recordings after delivering $(A)$ one distal nerve stimulus $(B)$ one proximal nerve stimulus $(C)$ paired proximal nerve stimuli with stimulus interval $2 \cdot 2 \mathrm{~ms}$ and (D) with stimulus interval $2.0 \mathrm{~ms}$ (in $C$ and $D$ the first proximal stimulus occurs at the beginning of the trace), (E) one distal and one proximal stimulus coupled to each other with the proximal stimulus delayed $11.6 \mathrm{~ms}$ and $(F)$ with the proximal stimulus delayed $11.4 \mathrm{~ms}$. Time bar $10 \mathrm{~ms}$. Further description in text.

value for all 10 motor units was $1.25 \pm 0.22 \mathrm{~ms}$ and for the 6 individual mean values $1.28 \pm 0.22 \mathrm{~ms}$.

In a few motor units the refractory period was also determined with test stimulus strength 100-300\% above the axonal threshold. The axonal refractory period then was $0 \cdot 0-0.2 \mathrm{~ms}$ shorter than at the $100 \%$ level. The shortest refractory period measured for one motor unit was $0.8 \mathrm{~ms}$.

THE REFRACTORY PERIOD AFTER A CONDITIONING ANTIDROMIC PROPAGATED NERVE IMPULSE

For the 28 accessory nerve motor units in this study, the axonal refractory period measured at low test stimulus strength after a conditioning antidromic impulse ranged from 1.2 to $2.8 \mathrm{~ms}$. In only one motor unit was it longer than $2.5 \mathrm{~ms}$. In five motor units it was shorter than $1.5 \mathrm{~ms}$. The mean value for all 28 motor units was $1.80 \pm 0.36 \mathrm{~ms}$ and for the seven individual mean values $1.83 \pm 0.26 \mathrm{~ms}$. Corresponding data from the ten reinnervated motor units were within the same range. When the results obtained in the accessory nerve motor units were compared with the results obtained in reinnervated motor units of this and the previous study, ${ }^{5}$ no statistically significant difference was found.

Figure 2 shows the individual mean values of the axonal conduction velocities and axonal refractory periods using low test stimulus strength for each of the 10 subjects in this study together with corresponding data from a previous study, ${ }^{5}$ that is, in total from 22 subjects and from 109 motor units. The mean value of the 22 individual mean values was for the refractory period $1.70 \pm 0.26 \mathrm{~ms}$ and for the 


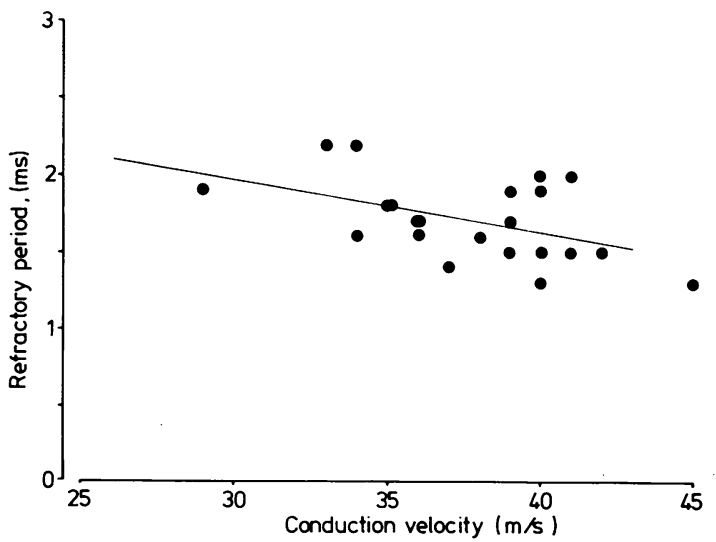

Fig 2 The individual mean values of the axonal conduction velocities and the axonal refractory periods after an antidromic propagated nerve impulse using low test stimulus strength of 22 healthy subjects in whom 109 motor units were investigated. Regression line marked as illustration. Further description in text.

conduction velocity $37 \cdot 7 \pm 3.6 \mathrm{~m} / \mathrm{s}$. An inverse relationship between these parameters was present (correlation coefficient $-0.47, \mathrm{p}<0.05$ ) as previously reported. ${ }^{5}$

For the 10 accessory nerve motor units studied with high test stimulus strength, the axonal refractory period after an antidromic propagated impulse ranged from 0.4 to $1.2 \mathrm{~ms}$. The mean value was 0.88 $\pm 0.26 \mathrm{~ms}$. This was significantly longer $(\mathrm{p}<0.05)$ than the corresponding value of the previous study ${ }^{5}$ $(0.52 \pm 1.18 \mathrm{~ms})$ when no correction for the stimulus spread at the test point was made ${ }^{5}$ (cf Methods).

Stimulus spread at the distal stimulus point might inadvertently make the calculated axonal refractory period after an antidromic propagated nerve impulse too short. Therefore the distal stimulus strength should be low. In this study it was $10 \%$ above the axonal threshold at rest to be safely suprathreshold. In five experiments attempts were made to minimise the stimulus spread by using needle electrodes inserted as near to the nerve as possible and delivering stimuli as close as possible to the axonal threshold at rest. This made no difference in two motor units but reduced the calculated refractory period by $0.2 \mathrm{~ms}$ in two motor units and by 0.4 $\mathrm{ms}$ in another (cf Discussion).

\section{COMPARISON OF THE RESULTS OBTAINED BY \\ EACH METHOD}

In all 37 motor units the axonal refractory period was determined using low test stimulus strength both after a conditioning electrical nerve stimulus and an antidromic propagated nerve impulse. In only one motor unit were the results the same. In the other 36 motor units the refractory period was longer when measured after an electrical nerve stimulus than after an antidromic nerve impulse. The difference ranged from $0 \cdot 1$ to $1 \cdot 1 \mathrm{~ms}$. For most of the motor units the difference was $0.4-0.8 \mathrm{~ms}$, for six motor units it was less than $0.4 \mathrm{~ms}$ and for five motor units it was more than $0.8 \mathrm{~ms}$. The mean value of the difference between the results with each method was $0.54 \pm 0.29 \mathrm{~ms}$.

For 10 motor units also studied using high test stimulus strength, the difference between the results obtained by each method was of the same magnitude as observed with low test stimulus strength, ranging from 0.2 to $0.8 \mathrm{~ms}$ and with a mean value $0.41 \pm 0.27 \mathrm{~ms}$.

\section{Discussion}

Apart from this present and previous studies, ${ }^{5710}$ no other systematic studies of the refractory period of single human alpha motor axons have been reported. The results are in good agreement with what is known about the refractory period of medullated nerve fibres in animals. ${ }^{11}$

The axonal refractory period in the present study was $1.28 \pm 0.22 \mathrm{~ms}$ using paired electrical stimuli and high test stimulus strength. This is longer than the corresponding values in human never trunk studies in the arm: $0.7 \pm 0.18 \mathrm{~ms}$ was reported by Kimura et al for the motor nerve fibres in the ulnar nerve ${ }^{4}$ and $0.6-0.7 \mathrm{~ms}$ was reported by Gilliatt et al for the mixed nerve fibre population in the median nerve. ${ }^{2}$ The main reason for this is probably the fact that the peroneal nerve fibres have lower conduction velocities and that the refractory period is inversely related to the conduction velocity. ${ }^{511}$ However, it should also be pointed out that the electromyographic techniques used here are unfavourable to the identification of motor units with the highest axonal conduction velocities. ${ }^{8}$ Another reason is that the temperature of the lower leg is normally lower than that of the arm, and that the refractory period is longer at low temperature. ${ }^{512}$ In the present study the skin temperature was $32^{\circ}$ while in the study by Gilliatt et al it was $35^{\circ}$. Finally, higher stimulus strength in the nerve trunk studies may also be a contributory factor.

The previous study of the axonal refractory period after an antidromic propagated nerve impulse was mainly made on reinnervated motor units. ${ }^{5}$ The results were now confirmed in normal motor units of the short toe extensor muscle. When measured with low test stimulus strength the refractory period was the same and exhibited the same relationship to the 
axonal conduction velocity. When measured with high test stimulus strength the refractory period was longer in this study since correction for test stimulus spread was made, as anticipated in the previous study. ${ }^{5}$

The high test stimulus strength used in this study, $100 \%$ above the axonal threshold at rest, is probably not high enough to determine the absolute refractory period before a propagated impulse can be elicited, but a few experiments with higher test stimulus strength indicated that the results at the $100 \%$ level are not far from the absolute refractory period. This is also indicated by the slope of the excitability changes described in a previous study. ${ }^{5}$ Moreover, it must be pointed out that higher stimulus strength is not acceptable when patients are investigated, owing to pain caused.

The low test stimulus strength used in this study, $10 \%$ above the axonal threshold at rest, was suitable in order to be sure that the stimulus was suprathreshold. The total length of the relative refractory period is probably only slightly longer than measured in this way judging from the slope of excitability changes and the recovery of conduction velocity as described previously. ${ }^{7}$ With lower test stimulus strength a greater number of stimuli are necessary to determine whether blocking is due to refractoriness or subthreshold stimulation and this is a drawback especially when patients are investigated.

There was a systematic difference between the results obtained by each of the methods used here: the axonal refractory period after a conditioning electrical nerve stimulus was always longer, in mean $0.5 \mathrm{~ms}$, than that after an antidromic propagated nerve impulse. The conduction velocity and excitability changes are known to be the same for orthoand antidromic propagation. ${ }^{13}$ However, the conduction distance for the ortho- and the antidromic impulses might be different: while the stimulus spread at each stimulus point is balanced when the latency difference is calculated, the stimulus spread at each stimulus point will be summated when the shortest time interval without blocking of the antidromic propagated impulse is measured. Thus, this time interval will be shorter due to shorter conduction distance and the calculated refractory period will inadvertently be too short. However, when the stimulus spread was minimised, the axonal refrac- tory period after an antidromic impulse was still shorter than after an electrical nerve stimulus. Different properties of the nerve fibre membrane and the surrounding tissues after an electrical shock and after an antidromic propagated nerve impulse might play a role. To elucidate this requires animal experiments (Borg, Tegnér in preparation). For studies of pathological states both methods seem equally reliable when proper normal data are available.

\section{References}

' Tasaki I. Nervous Transmission. Thomas Springfield 1953.

${ }^{2}$ Gilliat RW, Willison, RG. The refractory and supernormal periods of the human median nerve. $J$ Neurol Neurosurg Psychiatry 1963;26:136-47.

${ }^{3}$ Lowitzsch K, Hopf HC. Refraktärperiode und Übermittlung frequenter reizserien im gemischten peripheren Nerven des Menschen. J Neurol Sci 1972;17:255-70.

${ }^{4}$ Kimura JA. Method for estimating the refractory period of motor fibres in human peripheral nerve. $J$ Neurol Sci 1976;28:485-90.

${ }^{5}$ Borg J. Axonal refractory period of single short toe extensor motor units in man. J Neurol Neurosurg Psychiatry 1980;43:1:917-24.

6 Hopf HC. Untersuchungen über die Unterschiede in der Leitgeschwindigkeit motorischer Nervenfasern beim Menschen. Dtsch Z Nervenheilk 1962;183:579-88.

${ }^{7}$ Borg J. Effects of prior activity on the conduction in single motor units in man. $J$ Neurol Neurosurg Psychiatry 1983;46:317-21.

${ }^{8}$ Borg J, Grimby L, Hannerz J. Axonal conduction velocity and voluntary discharge properties of individual short toe extensor motor units in man. J Physiol (Lond) 1978;277:143-52.

${ }^{9}$ Borg J. Properties of single extensor digitorum brevis motor units in elderly man. Muscle Nerve 1981;4:429-34.

${ }^{10}$ Borg J. Axonal refractory period of single short toe extensor motor units in neuropathies and neuromuscular diseases. J Neurol Neurosurg Psychiatry 1981;44:1136-40.

"Paintal AS. Conduction in mammalian nerve fibres. In: Desmedt, ed. New Developments in Electromyography and Clinical Neurophysiology, vol. 2, Basel: Karger, 1973;19-41.

12 Paintal S. Effects of temperature on conduction in single vagal and saphenous myelinated nerve fibres of the cat. J Physiol (Lond) 1965;180:20-49.

${ }^{13}$ Adrian ED. The recovery process of excitable tissues. Part II. J Physiol (Lond) 1921;55:193-225. 